\title{
Concrete Crack Detection using Relative Standard Deviation for Image Thresholding
}

\author{
Tao Peng', Thathupara Subramanyan Kavya², Young-Min Jang², Byeong-Woo Kim ${ }^{3}$ \\ Department of Electrical Engineering, University of Ulsan, Republic of Korea. \\ ${ }^{1}$ Master, Students, School of Electrical Engineering, University of Ulsan, The Republic of Korea. \\ ${ }^{2}$ Ph.D., School of Electrical Engineering, University of Ulsan, The Republic of Korea. \\ ${ }^{3}$ Professor, School of Electrical Engineering, University of Ulsan, The Republic of Korea.
}

\begin{abstract}
:
One of the biggest challenges with the reinforced concrete structures and concrete buildings are to identify the possible cracks. Cost-effective automated structural maintenance systems have attracted considerable attention in the recent past to detect the cracks in concrete structures. In this paper, we propose a concrete crack detection system based on image processing technique. For pre-processing an image, the anisotropic diffusion process is used and the top-hat and bottom-hat filtering techniques are used to enhance the contrast of an image. Top-hat filter enhances bright objects of interest from a dark background and the bottom-hat filter does the opposite. The existing algorithms require manual calibration of the threshold of some predefined specific parameters. However, we are using a new strategy for threshold selection based on relative standard deviation; this makes a difference in the image segmentation. The proposed method is tested with almost 200 images from public datasets and the experimental results prove that this method is efficient and effective to detect cracks in the concrete.
\end{abstract}

Keywords: Crack Detection, Image Processing, Image Segmentation, Thresholding.

\section{INTRODUCTION}

One of the major constraints in reinforced concrete structures and buildings are that, it deteriorates over time. Cracking seems unavoidable and it appears in all types of concrete structures such as beams, walls, bridges, and slabs. These cracks give access to harmful and corrosive chemicals to penetrate the concrete structure, which consequently damages their integrity as well as esthetics. Timely and accurate monitoring of concrete structures are essential to detect cracks at an early stage which enables the maintenance of concrete structures and prevent accidents. However, this remains a challenging task due to the intensity of crack, uneven illumination, shadows, noise pollution, shading, blemishes or concrete spall in images.

Due to the late infrastructural developments in Korea, the percentage of concrete structures older than 30 years was estimated at $3.8 \%$ in 2014, while it is expected to increase exponentially and reach $13.8 \%$ in 2024 and $33.7 \%$ in 2029 [1] . An excellent crack detection system plays a major role in the maintenance of infrastructure. Globally, a large amount of money is spent to maintain different concrete structures which include repair, reconstruction and renewal to ensure safety. One of the traditional methods to detect cracks in concrete structures is to do a manual inspection. However, this method is very time consuming, more laborious and expensive. To overcome this limitation, researchers recently started working on improving the crack detection methods [2]. A single camera is used to collect the images with more accurate information and hence make the vision-based crack detection system as one of the most promising method.

A quick and reliable system to detect cracks by means of automated procedures replaces the slow and subjective manual inspection. In this paper, we are proposing a concrete crack detection method based on computer vision.

The main advantages of the proposed method are:

- Additional sensors are not required to detect cracks[3-4]

- Cost-effective, easy to implement, highly efficient, high level of accuracy.

- A new thresholding method based on the relative standard deviation for image segmentation.

The rest of the paper is organized as follows: In section 2, a brief review of the literature related to the image-based crack detection is presented Section 3 describes the proposed crack detection method. Experimental results are discussed in section 4 followed by the conclusion and some suggestions for future enhancements.

\section{RELATED WORK}

Automatic crack detection became an interesting topic for researchers in the recent years due to the advancement of technology. Manual crack identification is not feasible for all the applications due to practical difficulties like manual inspections and reliability of the record. In the last decade, researches invested a lot of time and effort in implementing automatic and non-automatic crack detection [5-7].

Byunghyun et.al [8] proposed a method for automated visionbased concrete crack detection using deep learning technique. Convolutional neural networks (CNN) is used in this technique. A well-known AlexNet is trained with images scraped from the internet for crack detection. A probability map is developed using a softmax layer value to add 
robustness to sliding window detection and a parametric study was carried out to determine its threshold. This method speeds up the detailed crack assessment with a good level of accuracy using vision devices such as cameras and drones.

Cao et.al proposed an autonomous concrete detection based on deep fully convolutional neural network (FCN) for semantic segmentation on concrete crack images [9]. Ah-Ram et.al [10] implemented a two-stage image processing pipeline method for crack segmentation based on deep learning image processing method. A deep learning based method for crack detection is proposed by Lei et.al [11]. Each image patch is classified with a trained deep convolutional neural network. Superior crack detection performance is provided by the combination of the learned deep features and the proposed deep learning framework.

The major limitation of all these methods is that it needs training for detecting all types of cracks. However, all the cracks associated cannot be included and trained, irrespective of the amount of training data set used.

Nhat-Duc [12] used gray intensity adjustment method to detect cracks based on improved Otsu method. The major limitation of this research work is that the users must fine-tune two factors such as adjusting ratio and margin parameter. However, in practical cases it may be difficult to calculate those values.

An edge-based crack detection using crack width transform is proposed by Hyunwoo et.al [13]. This technique consists of five steps. In the first step, the crack candidate pixels are identified and classified from the opposing edges and a width map is generated. An aspect ratio filtering is carried out in the second stage to remove the noise. Missing pixels are searched for and restored in the next two stages and in the final step, residual noise is removed based on image adaptive thresholding.

Zhong et.al [14] proposed an algorithm for concrete surface crack detection based on the genetic programming (GP) and percolation model. The cracks are pre-extracted in the first step using the image processing model of GP followed by extraction of crack skeleton and calculation of crack tip in the second step. High speed percolation having high precision is used to detect the cracks. Concurrently, to establish the connection, the fracture unit areas are scanned. The final step is to connect the pre-extracted cracks with the cracks detected by the percolation and the mass interference area is removed to obtain the real cracks within the concrete surface. A much improved method to detect concrete surface crack is implemented by Zhong et.al [15]. An improved pre-extraction and an additional percolation technique are used to process the images in this method.

\section{PROPOSED METHOD}

Safety is one of the major concerns in the construction industry and the proposed automatic concrete crack detection system plays a vital role to ensure that the standards. In this paper, we are presenting an effective crack detection method for concrete structures based on the image processing technique with a new thresholding approach. Fig. 1 shows the general architecture of the proposed method.

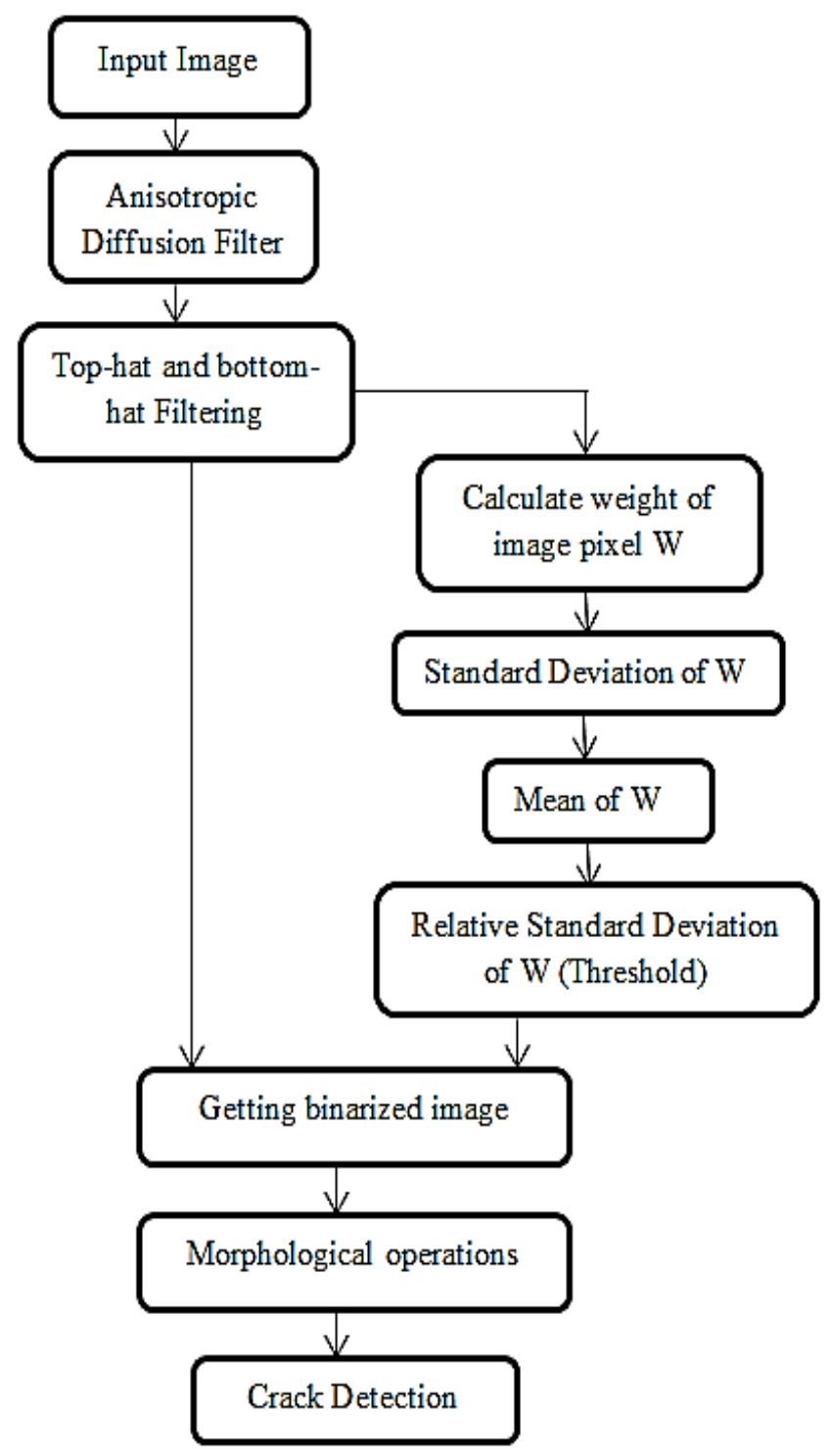

Fig.1. Flow chart of the proposed method

\section{III.I Anisotropic Diffusion Filter}

The first step in processing an image is to convert it into a grey scale image. The original image is pre-processed using an image-smoothing filter which removes the unnecessary noise. Anisotropic diffusion filtering method is used with the intention to reduce the noise without damaging the significant parts of the image content, typically the edges, lines and other details that are important for image processing applications [16-17].

Anisotropic diffusion generates a parameterized family of blurred images from a single image through a diffusion process. Every resulting image in the parameterized family is convolved with the two-dimensional Isotropic Gaussian filter where the parameter of the image is directly proportional to the width of the filter. Anisotropic diffusion is the generalization of a diffusion technique. The diffusion technique is a linear and space invariant transformation of the actual images. Each image in the family is a combination of 
the actual image and a filter that depends on the local content of the actual image.

A law of diffusion is applied on the pixel intensities to obtain smooth textures in an image. The purpose of the threshold function is to avoid diffusion across the edges and hence the edges are preserved. The edges are then used to segment the image without being disturbed by the noise.

\section{III.II Image Enhancement}

Image resolution and contrast are the major factors which determines the accuracy of the image segmentation. Some of the characteristics of the concrete surface images are a complex background, uneven light reflections and illumination which may have an influence on the final detection results. Top-hat and bottom-hat filtering technique is used in this research work to enhance the contrast of the image. The bright objects which are shorter than the structuring element are retrieved by the top-hat filter and the dark elements by the bottom-hat transform filter. The enhanced image is produced by adding the results of the tophat filter and subtracting the results of the bottom-hat filter from the original image [18]. Contrast enhancement improved the object perceptibility by enhancing the difference in the brightness between the object and the background. The equations for top-hat, bottom-hat and enhanced image calculation are as shown below.

$$
\begin{gathered}
I_{\text {top }}=I-(I \circ S) \\
I_{\text {bot }}=(I \bullet S)-I \\
I_{\text {enhance }}=I+I_{\text {top }}-I_{\text {bot }}
\end{gathered}
$$

Where ' $\mathrm{I}$ ' is the input image and ' $\mathrm{S}$ ' represents the structuring element. Equation (1), (2) and (3) represents the top-hat, bottom-hat and enhanced image respectively.

\section{III.III Threshold Selection Based on Relative Standard Deviation}

An optimal threshold value plays an important role in detection the cracks with at most accuracy. Most of the existing algorithms use a fixed value of threshold or manual calibration of threshold in terms of some predefined specific parameters. However, this may not provide good results for all practical images. In this paper, we propose a new method for binarization based on relative standard deviation as a new threshold for crack detection of an image.

The new thresholding approach automatically calculate the threshold values based on the input image. We calculate threshold value by calculating the gradient weight of an image pixel followed by the mean value and standard deviation of the gradient weight. Finally, the relative standard deviation (RSD) is calculated and the threshold value is applied to each pixel of an image. Each pixel value is compared with the calculated threshold value and if the pixel value is higher than the threshold value, the out pixel becomes zero otherwise one.
The relative standard deviation is a normalized measure of the dispersion of a probability distribution. This is defined as the standard deviation to the mean ratio. The motivation behind using relative standard deviation is because, it has the ability to detect shadow from an image [19]. Relative standard deviation is a physical measure of the sensitivity of an image and is more informative than the standard deviation.

Let RSD is the relative standard deviation then, the proposed condition for concrete crack detection at each pixel I (i, j) is given as

$$
D=\left\{\begin{array}{rr}
1, & \text { if } I(i, j)>R S D \\
0, & \text { otherwise }
\end{array}\right.
$$

Where $I(i, j)$ is the contrast-enhanced image.

This is then followed by a morphological operation to remove the noisy pixels and intact objects from the segmented image.

This is the most simple and effective technique for binary conversion. We have tested our algorithm on a wide range of images taken at different conditions and are able to get accurate crack detection results. Another advantage of this algorithm is that it doesn't need any training or learning phases.

\section{Proposed algorithm}

Various steps of the proposed algorithm are as follows:

Step 1: Transformation of the color image into gray scale image.

Step 2: Perform anisotropic diffusion for edge-preserving smoothing.

Step 3: Perform the image contrast enhance using top-hat and bottom-hat filtering.

Step 4: Calculate the gradient weight of the enhanced image W.

Step 5: Calculate the mean and standard deviation of W.

Step 6: Compute relative standard deviation based on step 5.

Step 7: Apply binary morphological operation on crack detected image to remove the unwanted noise.

\section{III.IV Image Dataset for Concrete Crack Detection}

A group of researches collected various concrete crack images from Middle East Technical University campus buildings*. The database contains a total of 40,000 RGB images $(20,000$ positive images and 20,000 negative images) with a resolution of 227 x 227 . For segmentation, we have randomly selected 100 images with crack and 100 images without cracks from a 20,000 cracked and 20,000 non-crack images respectively. Some of the sample images are shown in Fig.2.

*http://dx.doi.org/10.17632/5y9wdsg2zt.1 

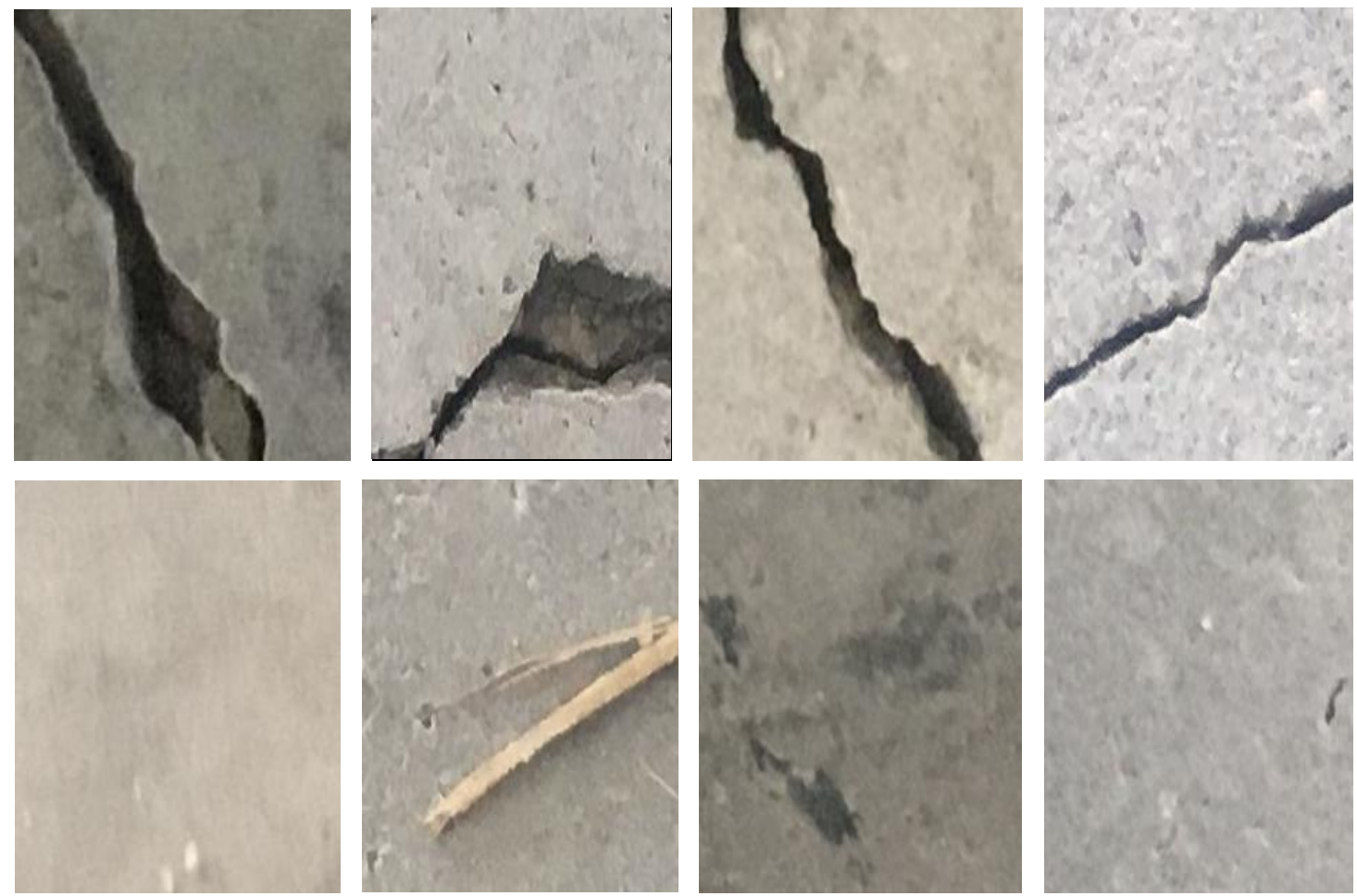

Fig 2: Positive and negative sample images from the database

\section{EXPERIMENTAL RESULTS}

The proposed algorithm has been applied to several benchmark images. For evaluating the proposed crack detection method, we consider a publicly available concrete crack data set. The proposed algorithm was implemented in MATLAB R 2018b and the performance is evaluated in terms of area, perimeter, precision, recall and F-measure.
To compare the new thresholding method, we tested the same images binarized with one of the prominent thresholding methods such as Otsu's threshold [20-22] as shown in Fig.3. (b). However, with different illumination and surface structure, this thresholding method does not provide better results. In contrast, our new thresholding method has given reasonably good results as shown in Fig.3. (c).
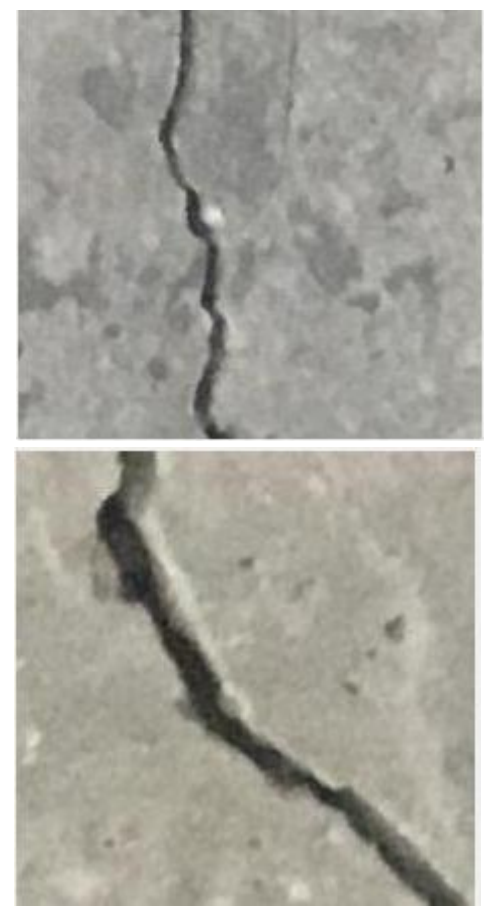
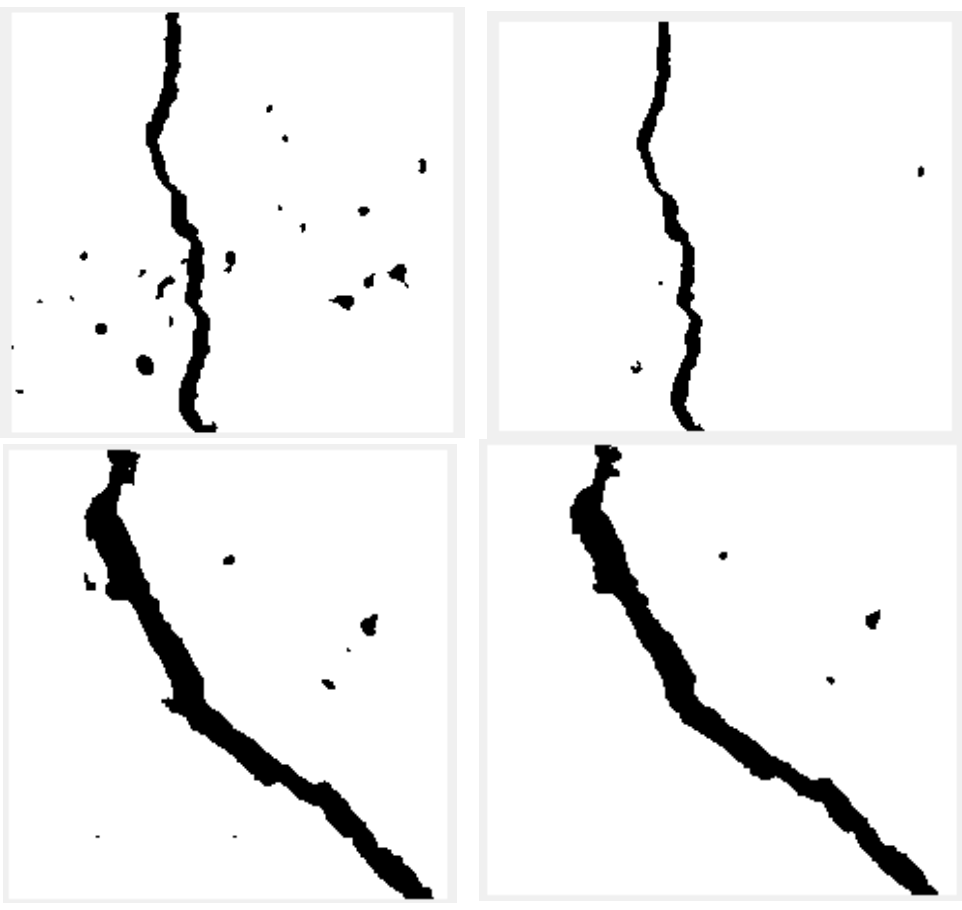

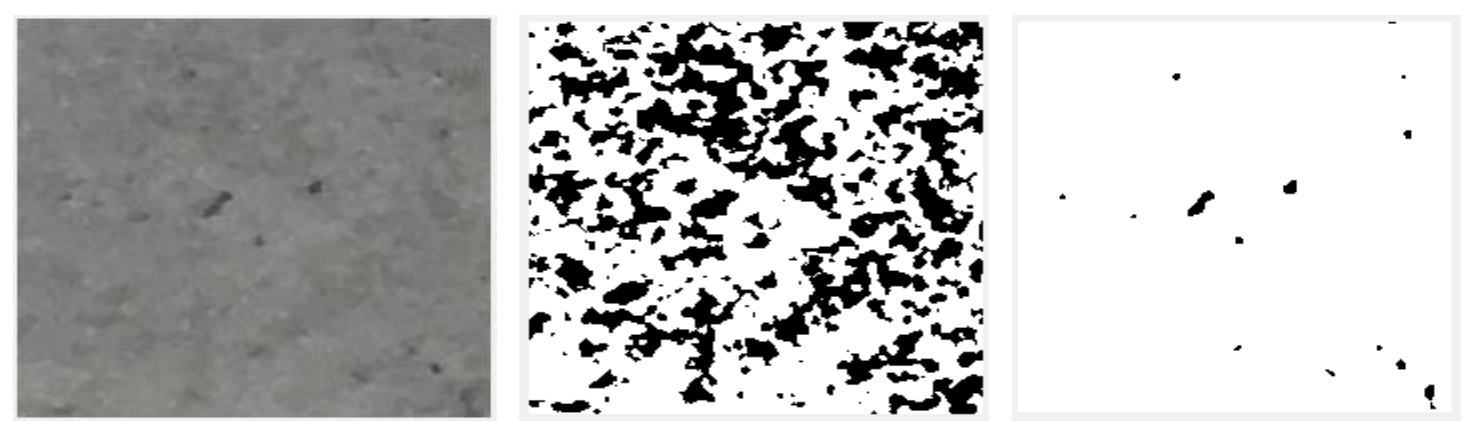

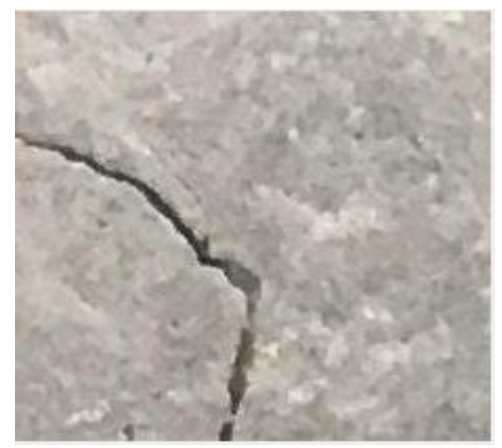

(a)

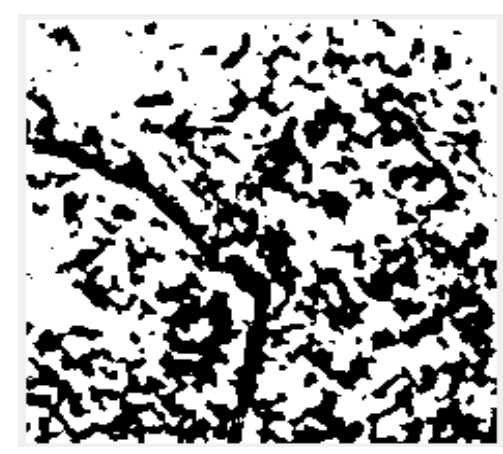

(b)

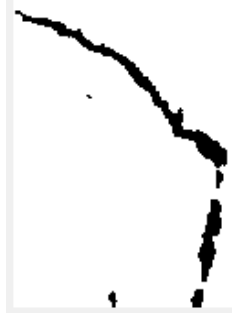

(c)

Fig :3.a) Original image b) Otsu threshold c) proposed method before applying the morphological operation

The results demonstrate that the new thresholding can significantly improve the efficiency and accuracy of the image segmentation compared with the standard thresholding method. The new method can segment the images which has different surface structures and illuminations etc. The first two images are ideal images where the Ostu's thresholding manages to provide better results whereas the third and fourth images has different surface structure and illumination. It is clear from the experimental results that Ostu's method failed to generate an acceptable result. However, our new thresholding shows the significant improvement in segmentation results in all different type of images. It is shown in Fig.3(c).

We tested this new thresholding technique on around 200 images from the public data set of concrete crack detection and in all the cases, the results are satisfactory. Some of the experimental results are shown in Fig.4.
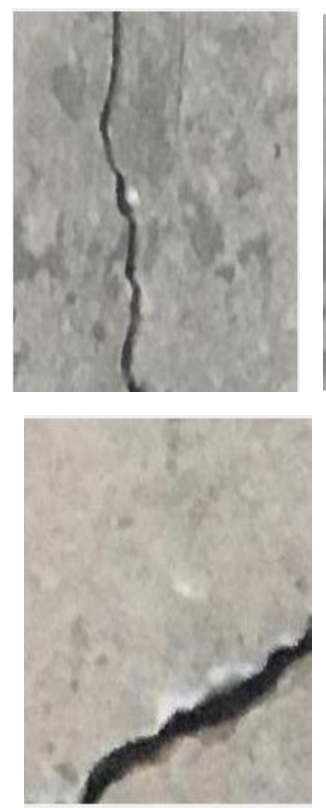
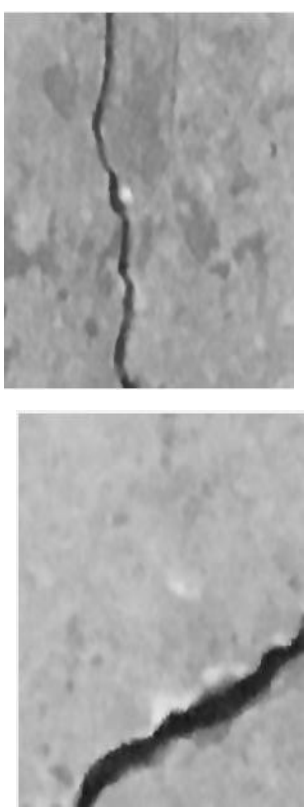
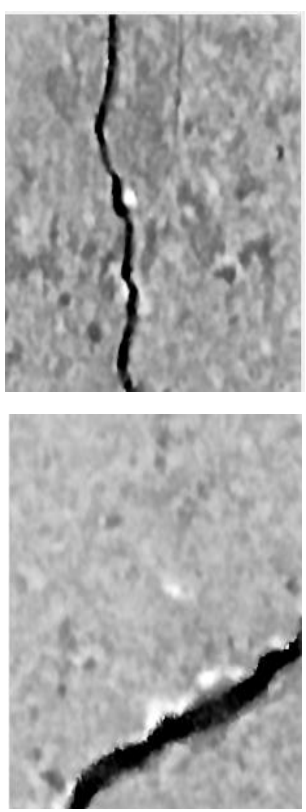
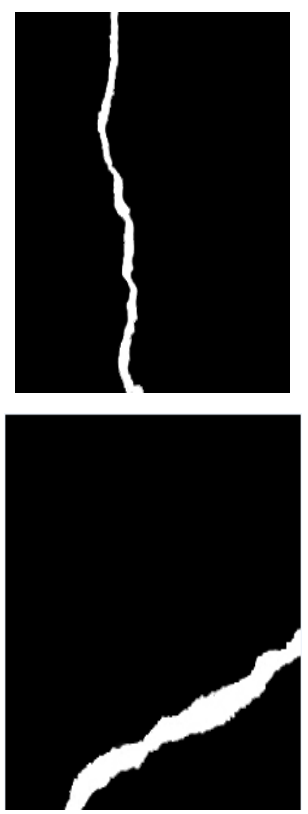
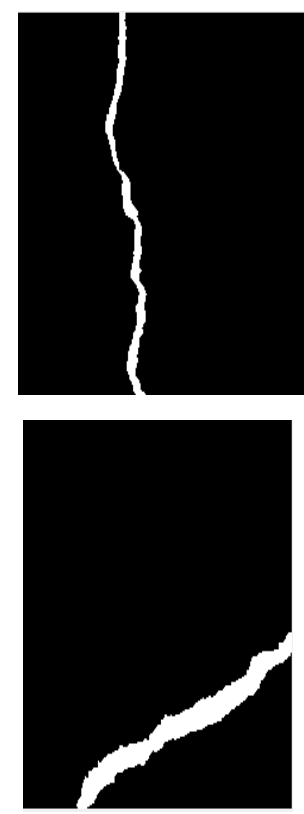

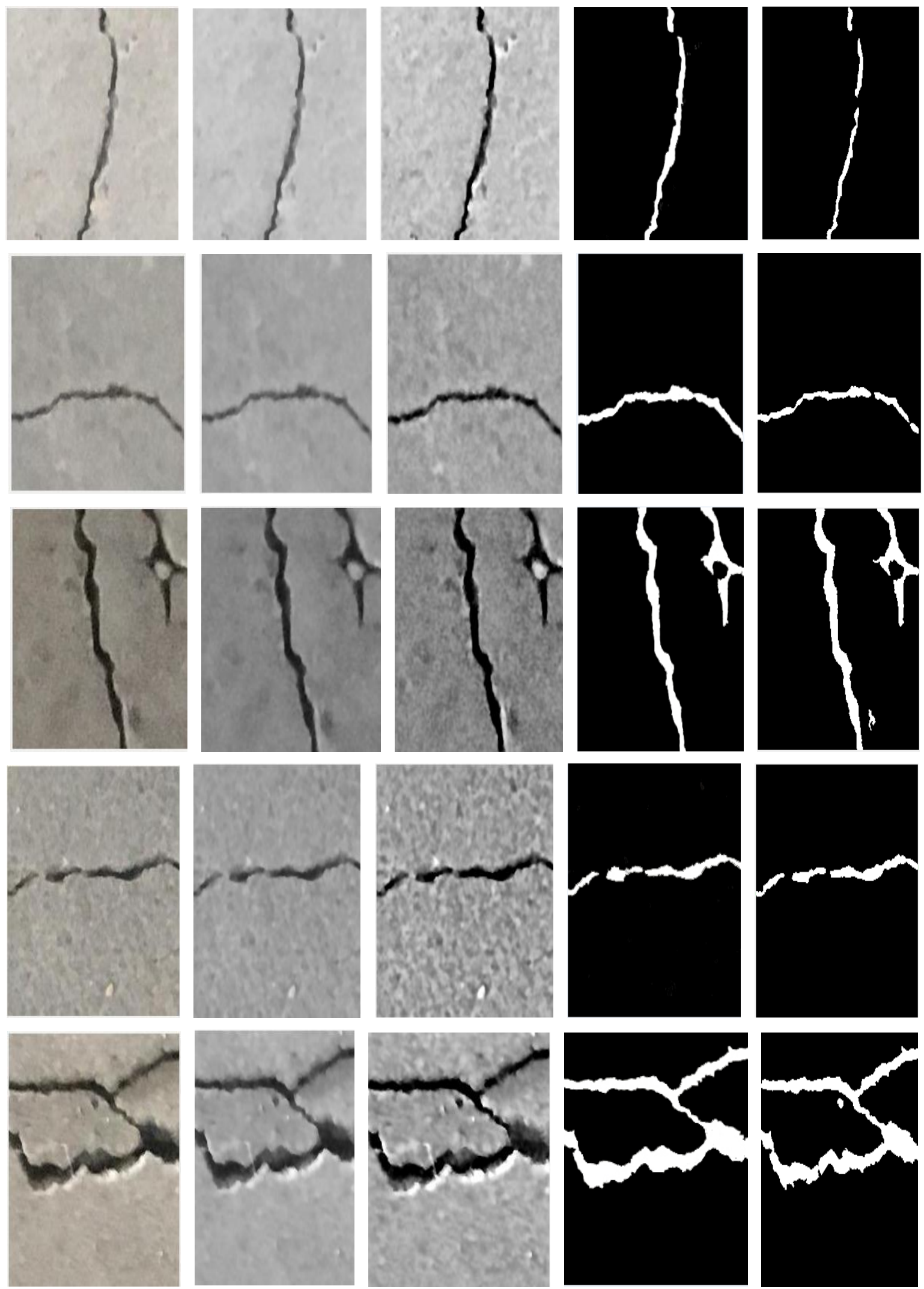


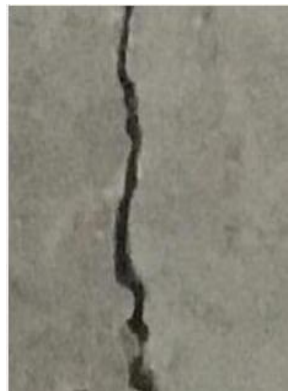

(a)

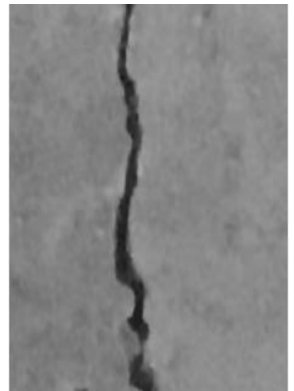

(b)

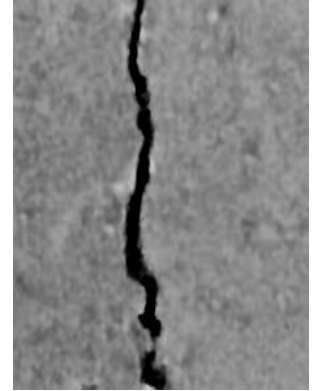

(c)

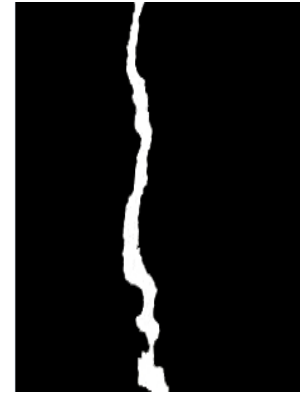

(d)

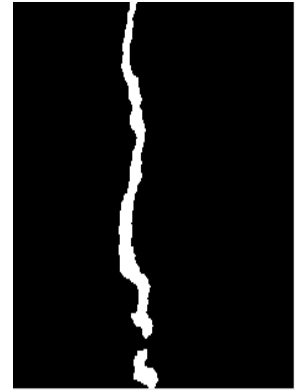

(e)

Fig 4: a) original images (image1-8) b) smoothed image c) contrast enhancement d) ground truth image e) segmented image

The cracked and non-cracked pixels can be classified from the binary segmented image using a simple thresholding. Finally, the boundary of the cracked regions and the detected boundaries are marked in the original image. This is shown in Figure.5 these results show the accuracy of the proposed method to detect the concrete crack from an image.
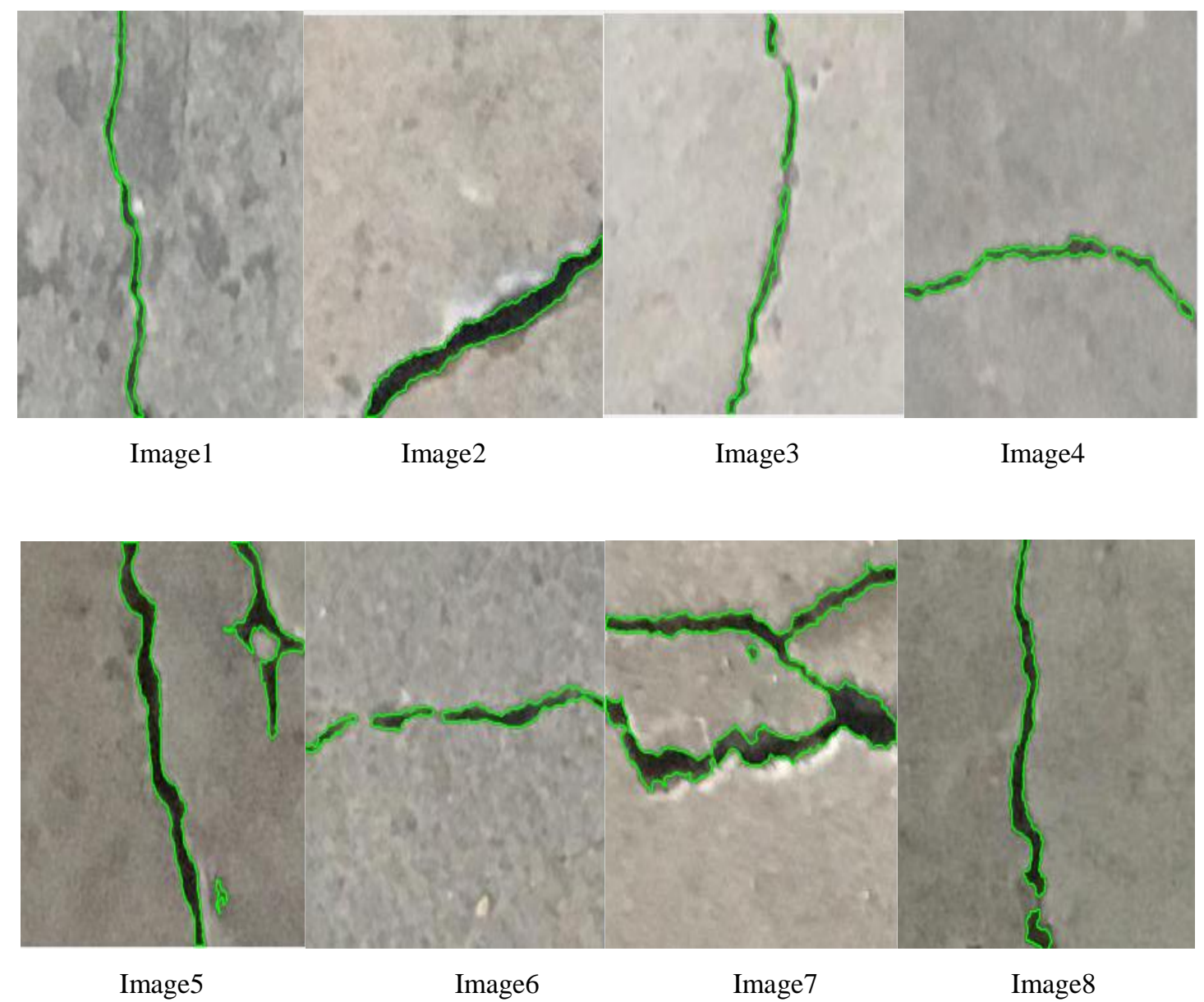

Fig 5: Crack detection results within the original image

The proposed crack detection system is evaluated using some parameters like area, perimeter, precision, recall and Fmeasure. These parameters have a significant role in the performance evaluation of the crack detection algorithm. The area of crack in an object is the total number of pixels located within the object boundary. The crack perimeter is calculated with the number of pixels on the boundary of the object. This is tabulated in Table 1 . 
Table 1. Result of crack analysis

\begin{tabular}{|c|c|c|}
\hline Test Image & Area & Perimeter \\
\hline Image1 & 1344 & 609 \\
\hline Image2 & 2725 & 582 \\
\hline Image3 & 1162 & 572 \\
\hline Image4 & 1356 & 618 \\
\hline Image5 & 4405 & 1181 \\
\hline Image6 & 1531 & 583 \\
\hline Image7 & 6459 & 1602 \\
\hline Image8 & 2043 & 638 \\
\hline
\end{tabular}

Precision and recall measure the exactness and completeness of segmentation respectively and F-measure is the harmonic mean of precision and recall. Higher the F-measure, better the performance is

$$
\begin{aligned}
& \text { Precision }=\frac{T P}{T P+F P} \\
& \text { Recall }=\frac{T P}{T P+F N} \\
& F-\text { measure }=\frac{2 * \text { Precision } * \text { Recall }}{\text { Precision }+ \text { Reacll }}
\end{aligned}
$$

Pixels labeled as crack pixels in the ground truth are correctly recognized as crack pixels are denoted as true positive (TP) similarly the non-crack pixels in the ground truth are incorrectly recognized as crack pixels denoted as false positives (FP) and pixels labeled as crack pixels in the ground truth are incorrectly detected as non-crack pixels are denoted as false negatives (FN). We used around 200 images from the database to evaluate the performance. The average precision, recall and F-measure of our proposed algorithm are $93 \%, 80$ $\%$, and $86 \%$ respectively.

\section{CONCLUSION}

This paper presents an efficient and effective crack detection algorithm. The images are pre-processed with the help of anisotropic diffusion. These images are then enhanced using top-hat and bottom-hat filtering method. Finally, a new method of thresholding based on relative standard deviation is applied for segmentation. Around 200 images from a public data set are considered to evaluate the performance of the algorithm. The performance evaluation parameters like area, perimeter, precision, recall and F-measure are calculated. The average precision is around $93 \%$ and this value is greater than the recall $(80 \%)$ which indicates that, non-crack pixels are not detected incorrectly as crack pixels. Therefore, this method is efficient for the structural maintenance for construction engineers and building management agencies.

The proposed method is flexible, less complex, and costeffective. Future work leads a way to the concrete crack detection from real-time videos, improve the pre-processing method to reduce false detections and incorporate classifier to improve surface crack detection without much overheads.

\section{ACKNOWLEDGEMENTS}

This work was supported by Korea Hydro \& Nuclear Power company through the project "Nuclear Innovation Center for Haeoleum Alliance" and "Industrial technology infrastructure building innovation project" through the Korea Institute for Advancement of Technology (Grant Number: P0014572).

\section{REFERENCES}

[1] Park C.H et al., "Future Trend of Capital Investment for Korean Transportation Infrastructure". Construction and Economy Research Institute of Korea ,Seoul ,Korea. 2016.

[2] Zhang W et al., "Automatic Crack Detection and Classification Method for Subway Tunnel Safety Monitoring ". Sensors. 14,2014.

[3] Pour-Ghaz $\mathrm{M}$ et al., "Wireless Crack Detection in Concrete Elements Using Conductive Surface Sensors and Radio Frequency Identification Technology", Journal Of Materials In Civil Engineering. 26(5):923929 May 2014

[4] Kuang KSC et al.,. "Crack detection and vertical deflection monitoring in concrete beams using plastic optical fibre sensors". Measurement Science And Technology, 14:205-216, 2003

[5] Mohan et al.,. "Crack detection using image processing: A critical review and analysis ". Alexandria Engineering Journal,57:787-798,2018.

[6] Xue-jun et al., "Crack detection of reinforced concrete bridge using video image ". Journal of Central South University,20:2605-2613,2013.

[7] Lins RG et al., "Automatic Crack Detection and Measurement Based on Image Analysis" IEEE Transactions On Instrumentation And Measurement, 65(3),2016.

[8] Byunghyun Kim , Cho S. "Automated Vision-Based Detection of Cracks on Concrete Surfaces Using a Deep Learning Technique ". Sensors, 18,2018.

[9] Dung CV et al., "Autonomous concrete crack detection using deep fully convolutional neural network ". Automation in Construction,99:52-58,2018.

[10] Ah-Ram Kim et al.,. "Crack Detection of Concrete Structure Using Deep Learning and Image Processing Method in Geotechnical Engineering [J]". Journal Of The Korean Geotechnical Society,34:145-154, Dec 2018.

[11] Zhang L et al., "Road Crack Detection Using Deep Convolutional Neural Network ". 2016 IEEE International Conference on Image Processing (ICIP); Phoenix, AZ, USA,2016.

[12] Hoang N-D et al., "Detection of Surface Crack in Building Structures Using Image Processing Technique with an Improved Otsu Method for Image Thresholding 
". Advances in Civil Engineering,2018.

[13] ChO $\mathrm{H}$ et al.," Image-Based Crack Detection Using Crack Width Transform (CWT) Algorithm ". IEEE Access, 6:60100-60114,2018.

[14] Ahong Qu et al., "The Algorithm of Concrete Surface Crack Detection Based on the Genetic Programming and Percolation Model ". IEEE Access,7:5759257602,2019.

[15] Zhong Qu et al., "Concrete surface crack detection with the improved pre-extraction and the second percolation processing methods ". PLOS ONE. July 2018.

[16] Oliveira H et al., "Automatic Crack Detection On Road Imagery Using Anisotropic Diffusion And Region Linkage ". 18th European Signal Processing Conference (EUSIPCO-2010); Aalborg, Denmark,2010.

[17] Hajiabolimr et al., "An Anisotropic Fourth-Order Diffusion Filter for Image Noise Removal". Int J Comput Vis.,92:177-191,2011.

[18] Ramasamy U et al., "Image Enhancement of Medical Images Based on an Efficient Approach of Morphological and Arithmetic Operations". International Journal of Computer Trends and Technology (IJCTT),49:253-258, July 2017.

[19] Khare $M$ et al.,. "Moving shadow detection and removal - a wavelet transform based approach". IET Computer Vision,8(6):701-17,2014.

[20] Priya M.S et al.,"Multilevel Image Thresholding using OTSU's Algorithm in Image Segmentation ". International Journal of Scientific \& Engineering Research. May -, 8(5):101-106,2017.

[21] Patil AB et al.,"OTSU Thresholding Method for Flower Image Segmentation ". International Journal of Computational Engineering Research (IJCER),06(05),2016.

[22] Vijay PP et al., "Gray Scale Image Segmentation using OTSU Thresholding Optimal Approach ". Journal for Research,02(05),2016. 